\title{
Residualidad de glifosato en suelos de Entre Ríos y su efecto sobre los microorganismos del suelo
}

\author{
María A. Sterren ${ }^{\bowtie}$; Walter Uhrich \& Silvia Benintende \\ Facultad de Ciencias Agropecuarias UNER.
}

\begin{abstract}
Resumen. El glifosato es un herbicida de amplio espectro, no selectivo, post emergente, muy usado en agricultura, pero del cual aún desconocemos sus impactos sobre la comunidad microbiana del suelo. Nuestro objetivo fue evaluar el efecto de la residualidad del glifosato sobre variables biológicas en suelos Vertisol y Molisol de Entre Ríos, a los 2 y 28 días posteriores a su aplicación, en condiciones de laboratorio. Para ello se incubaron muestras de suelo a $25^{\circ} \mathrm{C}$ con una dosis de glifosato de $2.14 \mathrm{~L}$ ingrediente activo/ha. Las variables biológicas fueron la actividad respiratoria (AR), el carbono de la biomasa microbiana (CBM), el cociente metabólico $\left(\mathrm{qCO}_{2}\right)$ y el potencial de mineralización de nitrógeno por incubaciones anaeróbicas (PMN-IA). También se cuantificó la residualidad del glifosato y del ácido aminometilfosfónico (AMPA). Se realizó un ANOVA para evaluar la interacción entre tratamiento y suelo para las variables CBM y PMN-IA. Los resultados mostraron que el glifosato tuvo baja residualidad en ambos suelos, y que su concentración disminuyó a los 28 días de su aplicación. Sin embargo, los contenidos de AMPA fueron altos durante todo el período estudiado. La AR, el CBM y el PMN-IA aumentaron en aquellos suelos con glifosato, lo que demostró que los microorganismos presentaron un sistema enzimático capaz de degradarlo y que fueron estimulados por una fuente de C lábil. Los valores de $\mathrm{qCO}_{2}$ en los suelos con aplicación del herbicida mostraron cambios en la fisiología de los microorganismos, y a los 28 días en el Vertisol se asoció a mayores contenidos de CBM. Esto presupone que existirían distintos grupos microbianos involucrados. Este trabajo mostró que la aplicación del herbicida impactó de manera diferente en los dos suelos. En el Molisol, las poblaciones microbianas no fueron afectadas significativamente, y en el Vertisol las poblaciones aumentaron acompañando la mayor residualidad del herbicida.
\end{abstract}

[Palabras clave: carbono de la biomasa microbiana, cociente metabólico, residualidad, vertisoles, variables biológicas]

\begin{abstract}
Aвstract. Glyphosate residual activity and its effect on soil microorganisms of Entre Ríos. Glyphosate is a broad-spectrum, not selective, post-emergent herbicide, widely used in agriculture, but we do not yet know its impacts on soil microbial community. The objective of this work was to evaluate the effect of residual of glyphosate on soil biological variables in Molisol and Vertisol soils of Entre Ríos at 2 and 28 days after application, under laboratory conditions. For this, soil samples were incubated at $25{ }^{\circ} \mathrm{C}$ with application of glyphosate at a dose of $2.14 \mathrm{~L}$ of active ingredient/ha. The biological evaluated variables were respiratory activity (AR), microbial biomass carbon (CBM), metabolic quotient $\left(\mathrm{qCO}_{2}\right)$ and nitrogen mineralization potential by anaerobic incubations (PMN-IA). Residuality of glyphosate and aminomethylphosphonic acid (AMPA) were also quantified. To evaluate the interaction between treatment and soil was performed with an factor analysis (ANOVA) for CBM and PMN-IA. The results showed that glyphosate had a low residuality in both soils, reducing its concentration to 28 days of application. However, the contents of AMPA were high throughout the study period in both soils. AR, CBM and PMN-IA increased in those soils with application of glyphosate, which showed that microorganisms showed an enzymatic system to degradate it and were encouraged by a source of labile $\mathrm{C}$. The values of $\mathrm{qCO}_{2}$ in soils with herbicide application showed changes in the physiology of microorganisms and at 28 days in the Vertisol was associated with higher contents of CBM which presupposes that there were different microbial groups. This work showed that herbicide application impacted differently on the two soils. In Molisol microbial populations were unaffected and in Vertisol the contents of C of microbial populations were increased and associated with greater amount of residual herbicide.
\end{abstract}

[Keywords: glyphosate, vertisol, biological variables, microbial biomass carbon; metabolic quotient; residual effects]

Editor asociado: Gervasio Piñerio

msterren@fca.uner.edu.ar 


\section{INTRODUCCIÓN}

El glifosato es un herbicida de amplio espectro, no selectivo, post emergente ampliamente usado en agricultura. La gran mayoría de la soja cultivada actualmente es de tipo RR (genéticamente modificada y resistente al herbicida glifosato), y algunos maíces también poseen esta característica, lo cual ha llevado a incrementar las dosis totales utilizadas por año y la frecuencia de las aplicaciones de glifosato. Por ejemplo, en las rotaciones agrícolas modales bajo siembra directa en la provincia de Entre Ríos se utilizan anualmente, en promedio, ocho ó más litros de glifosato por hectárea. Se considera al glifosato como un herbicida que se adsorbe fuertemente a los suelos, por lo que a pesar de ser muy soluble tiene reducida movilidad. Vereecken (2005), cuando estudió la movilidad de plaguicidas en suelos, encontró que el glifosato se adsorbe a las arcillas y a la materia orgánica, y que para que se movilice debe haber macroporos y grietas. La persistencia de los herbicidas se mide según la $\mathrm{DT}_{50^{\prime}}$ que es la cantidad de días necesarios para que un plaguicida se reduzca a la mitad de la concentración inicial, y la $\mathrm{DT}_{90^{\prime}}$ que es la cantidad de días necesarios para que un plaguicida se reduzca al $90 \%$ de la concentración inicial. Según la PPDB (Pesticida Properties Database) elaborada por la Agriculture and Environment Unit de la University of Hertfordshire (Inglaterra), la persistencia del glifosato de $\mathrm{DT}_{50}$ a campo es 12 días y en laboratorio es 49 días (a $20^{\circ} \mathrm{C}$ ), mientras que la $\mathrm{DT}_{90}$ en laboratorio es 159 días (a $20^{\circ} \mathrm{C}$ ). Algunos autores también mencionan la persistencia de niveles elevados de glifosato medido a partir de ${ }^{14} \mathrm{C}$ a los 217 días de aplicado (Heinonen-Tanski 1989), y otros, como Feng y Thompson (1990), encontraron entre 6\% y $18 \%$ de los niveles iniciales del herbicida 360 días después de su aplicación.

El glifosato se degrada relativamente rápido en el suelo, lo cual depende en gran medida de la actividad microbiana. Los microorganismos del suelo pueden romper los enlaces C-P y C-N y dar como producto sarcocina o ácido aminometilfosfónico (AMPA), que luego puede ser degradado a tasas más lentas. Wiren-Lehr et al. (1997) mencionan que las tasas de mineralización del glifosato están relacionadas tanto a la actividad como a la masa de microorganismos; esto determina su permanencia en el suelo. En consecuencia, la aplicación de glifosato podría afectar la actividad de los microorganismos del suelo $\mathrm{y}$, por lo tanto, distintas variables biológicas del mismo (Biederbeck et al. 2005; Benintende et al. 2005). Los microorganismos están vinculados a muchas funciones que tienen una importancia directa en la producción y la sustentabilidad agrícola como, por ejemplo, la intervención en los ciclos biogeoquímicos de nutrientes, la mineralización de los restos orgánicos, la inmovilización y solubilización de minerales, la degradación de xenobióticos, etc. Entre las variables biológicas del suelo que el glifosato probablemente afecte podemos mencionar la actividad respiratoria (AR), que es uno de los principales metabolismos microbianos que ocurren en el suelo y es un indicador sensible a diferentes condiciones de manejo y uso agrícola, y el carbono y el nitrógeno de la biomasa microbiana (CBM y NBM), cuyos métodos de medición están suficientemente probados como para incluirlos entre los índices de rutina en la determinación de calidad de suelos (Turco et al. 1994). También están aquellas variables relacionadas a la mineralización de nutrientes, como el potencial de mineralización de $\mathrm{N}$ de los suelos por incubaciones anaeróbicas (PMN-IA) propuesto por Waring y Bremner (1964), y que también fueron estudiadas por Echeverría et al. (2000), Bollero (2000), Calviño y Echeverría (2003) y Benintende et al. (2005) como indicadores de calidad biológica del suelo.

En general, las variables biológicas antes mencionadas se relacionan directamente con procesos de mineralización e intensidad de los flujos de carbono y de energía. Anderson y Domsch (2010) plantean que cualquier impacto que afecte a los miembros de la comunidad microbiana debería detectarse a nivel de comunidad a través de un cambio en una actividad particular. El cociente metabólico $\left(\mathrm{qCO}_{2}\right)$ es uno de los indicadores que surge a partir de la actividad respiratoria realizada por los microorganismos en relación a la masa microbiana total por unidad de tiempo. Bittencourt Barreto et al. (2008) afirman que se puede usar el $\mathrm{qCO}_{2}$ para evaluar efectos ambientales y antropogénicos sobre la actividad de la biomasa microbiana.

Diversos estudios muestran que la aplicación de glifosato en las rotaciones agrícolas afecta la dinámica de las poblaciones microbianas, con consecuencias sobre la sustentabilidad de los sistemas agropecuarios. Los efectos pueden ser mínimos, transitorios o constantes en el tiempo de acuerdo a la historia de aplicación del herbicida. En condiciones de campo, si 
bien algunos estudios han descripto efectos pequeños o nulos del glifosato sobre la actividad microbiana (Ferreira et al. 2006), otros han encontrado efectos considerables. Wardle y Parkinson (1990) observaron que la presencia de glifosato en el suelo se relacionó con incrementos temporales de las poblaciones de bacterianas y con su actividad. También Alexa et al. (2009) mencionaron que la degradación del glifosato a AMPA y $\mathrm{CO}_{2}$ está relacionada a la actividad microbiana y a factores que afectan dicha actividad. Nakatani et al. (2014) no encontraron diferencias significativas sobre el NBM, pero el CBM aumentó hasta $24 \%$ en los suelos con aplicación de herbicidas convencionales (sojas no resistentes a glifosato) en comparación con aquellos en los que se usó glifosato (soja RR).

Diferentes autores mencionan que la susceptibilidad a la degradación del glifosato por los microorganismos está muy relacionada con la textura del suelo. Sparks (2003) menciona que la interacción del glifosato con los componentes orgánicos e inorgánicos del suelo puede brindar información clave para predecir y comprender su comportamiento en el ambiente. En ese trabajo, Sparks afirma que los constituyentes de la fase sólida del suelo (e.g., arcillas y óxidos de aluminio, hierro y manganeso) tienen efectos significativos en los procesos de adsorción-desorción debido a su gran superficie específica y a su reactividad. Lerch et al. (2008) mencionan que para reducir el impacto ambiental de los agroquímicos es esencial entender los mecanismos que afectan la adsorción y la degradación de los mismos. La formación de residuos no extractables, que resultan de una captura irreversible por un establecimiento químico y/o secuestro físico del agroquímico y sus metabolitos en la matriz órgano mineral del suelo, es el mayor impedimento para su completa degradación por los microorganismos. Al Rabaj y Schiavon (2005) afirman quela adsorción delos pesticidas juega un rol fundamental en su destino dado que condiciona su presencia en la solución del suelo y, por lo tanto, su disponibilidad para una posible degradación microbiana y / o disipación en el ambiente. Varios autores muestran cómo el tiempo de residencia de los herbicidas en el suelo depende, entre otros factores, de su retención en el suelo, y de qué manera está afectado por su composición mineralógica (óxidos y arcillas) (Piccolo et al. 1994; Gerritse et al. 1996; Morillo et al. 2002; Gimsing et al. 2004). La inmovilización en suelos ocurre por la adsorción del glifosato a los óxidos y arcillas, lo que lleva a la formación de complejos superficiales. En la Argentina, Pessagno et al. (2008) estudiaron la adsorción de glifosato en suelos de diferente composición mineralógica en función de su concentración y del pH del suelo, y demostraron la formación de complejos entre el glifosato y la superficie de las arcillas (i.e., goethita, caolinita, illita y montmorillonita). El contenido de arcillas en suelos, el tipo de arcilla y su capacidad de intercambio catiónico son parámetros importantes a la hora de evaluar la adsorción de glifosato en el suelo (Glass 1987; Dion et al. 2001; De Santana et al. 2006). Dos santos Afonso (2010) encontró en suelos de Santa Cruz que la secuencia de adsorción de glifosato para las distintas arcillas presentes en los suelos de la zona fue: geotita>caolinita $>$ illita>montmorillonita, y que dicha adsorción afectó su persistencia en el suelo. El grado de complejización resultó dependiente de la concentración y del pH de la suspensión.

La residualidad del glifosato en el suelo podría variar entre Molisoles y Vertisoles. Los Vertisoles están caracterizados por un alto contenido de arcillas montmorilloníticas, con elevada área superficial. Por esta razón tendrían mayor afinidad por el glifosato y lo dejarían menos disponible para que los microorganismos del suelo lo degraden. En estudios realizados en Vertisoles de Entre Ríos, Primost et al. (2012) encontraron que el glifosato en el suelo se degradó principalmente al metabolito AMPA, y que una fracción puede persistir entre aplicaciones. Además, ambos compuestos mostraron afinidad con la matriz del suelo, por lo que al ingresar a los cuerpos de agua permanecen asociados al material en suspensión y luego pasan a formar parte del sedimento de fondo. Sin embargo, hay pocas investigaciones que muestren resultados de la degradabilidad de este herbicida en suelos agrícolas con características vérticas. Gómez et al. (2009) mencionan que la composición mineralógica de los principales suelos de la pampa húmeda argentina, donde hay contenidos altos de arcilla, podrían contribuir a la adsorción de glifosato en la matriz del suelo con lo cual disminuiría su degradación por parte de los microorganismos.

Por lo tanto, existen evidencias de que la residualidad del glifosato y/o de sus metabolitos afectaría algunas de las actividades microbianas de mineralización, inmovilización, respiración, etc. que tienen lugar en el suelo. El objetivo de este trabajo fue evaluar el efecto de la residualidad del herbicida glifosato sobre algunas variables biológicas en suelos Vertisol y Molisol de 
Entre Ríos en dos momentos posteriores a la aplicación del herbicida bajo condiciones controladas de laboratorio.

\section{Materiales y Métodos}

\section{Diseño experimental y toma de muestras}

Se seleccionaron áreas de suelo homogéneas correspondientes a los dos órdenes más importantes de la provincia de Entre Ríos: Molisol (Argiudol ácuico, Serie Tezanos Pinto) y Vertisol (Peluderte árgico, Serie María Dolores) (Soil Survey Staff 2010). La ubicación del área de muestreo para el suelo Molisol fue $31^{\circ} 51^{\prime} 45.04^{\prime \prime} \mathrm{S}-60^{\circ} 38^{\prime} 45.80^{\prime \prime} \mathrm{O}$ y para el suelo Vertisol fue $32^{\circ} 12^{\prime} 18.11^{\prime \prime} \mathrm{S}-60^{\circ} 10^{\prime} 26.46^{\prime \prime} \mathrm{O}$. El Molisol de la Serie Tezanos Pinto pertenece a la familia "fina, mixta, térmica". Son suelos profundos, moderadamente bien drenados, con un epipedón oscuro, franco-arcillolimoso a franco-limoso y un horizonte argílico oscuro, franco-arcillo-limoso a arcillo-limoso. Posee horizonte Ap de 00-17 $\mathrm{cm}$ franco-arcillo-limoso, estructura granular y en bloques con un contenido de arcilla de $27.6 \%$, donde el porcentaje de arcillas del grupo montmorillonitas es menor a 50\%. El Vertisol de la Serie María Dolores pertenece a la familia "fina, levemente alcalina, térmica", de los Peludertes árgicos (Vertisol con gilgai y un B2 textural), con un epipedón francoarcillo-limoso y un horizonte B2 argílico arcillo-limoso con concreciones de calcáreo que aparecen entre los 60 y $70 \mathrm{~cm}$. Posee horizonte Ap 00-12 cm franco-arcillo-limoso con estructura granular friable en húmedo, presencia de barnices "humic skins" y con un contenido de arcilla de $47.22 \%$, donde más de $50 \%$ pertenece a la clase montmorillonita (Plan Mapa de Suelos 1998, 2001). Las secuencias de cultivos en cada área de suelo desde el año 2007 hasta el momento del muestreo se muestran en la Tabla 1. Ambos lotes presentaban historia con aplicación de glifosato durante el barbecho y aplicaciones de glifosato durante el desarrollo del ciclo del cultivo para aquellos resistentes a este herbicida (soja y maíz).

El muestreo de suelo se realizó en junio de 2013 por triplicado, entre las líneas de cultivos, a una profundidad de $0-15 \mathrm{~cm}$. Cada muestra estuvo compuesta por 20 submuestras. La manipulación y el almacenamiento de las muestras siguió la guía general para tratamiento de muestras de suelo para evaluaciones biológicas de calidad de suelos (ISO 1993). Se evaluó por separado el impacto de la aplicación de glifosato en el Molisol y en el Vertisol. Los tratamientos fueron suelo con y sin aplicación de glifosato: Vertisol Control (VC), Vertisol con Glifosato (VG), Molisol Control (MC) y Molisol con Glifosato (MG), y se realizaron 3 repeticiones por tratamiento y para cada momento de muestreo.

Los tratamientos con aplicación de glifosato se realizaron a partir de una formulación comercial de glifosato isopropilamonio al $48 \%$ $\mathrm{P} / \mathrm{V}$ de la sal isopropilamina de $\mathrm{N}$-fosfonometil glicina (35.6\% P/V de equivalente ácido). La dosis de ingrediente activo (i.a.) para los tratamientos con aplicación de glifosato fue de $2.14 \mathrm{~L}$ i.a./ha y corresponde a un volumen de $6.01 \mathrm{~L} / \mathrm{ha}$ de esta formulación comercial que es utilizada en promedio en el ciclo de un cultivo. Para el cálculo del volumen a aplicar en los tratamientos se tuvo en cuenta la densidad aparente de cada tipo de suelo (Molisol, $1.25 \mathrm{t} / \mathrm{m}^{3}$; Vertisol, $1.1 \mathrm{t} / \mathrm{m}^{3}$ ) y una profundidad de penetración en la capa arable de $5 \mathrm{~cm}$. La aplicación de glifosato se hizo por pulverización y se diluyó en agua destilada para mejorar su distribución en el suelo. La misma cantidad de agua fue asperjada para los tratamientos control. La aplicación se realizó por única vez antes del periodo de incubación, sobre las muestras de suelo conservadas previamente en heladera. Se colocaron 250 $\mathrm{g}$ de suelo homogenizado manualmente en recipientes plásticos cerrados, cuyas tapas tenían pequeñas perforaciones que permitieron el intercambio gaseoso. En el interior se introdujo un vaso con agua destilada para mantener la humedad atmosférica. Las muestras se incubaron en estufa a $25^{\circ} \mathrm{C}$ por triplicado para cada tratamiento y período de muestreo, totalizando 36 las muestras que fueron evaluadas por separado. Los muestreos y determinaciones se realizaron al inicio (antes de la incubación y sin aplicación del herbicida), a los 2 días y a los 28 días posteriores a la aplicación del herbicida.

Tabla 1. Secuencia de cultivos en las áreas de suelo Vertisol y Molisol.

Table 1. Sequence of crops in the areas of Vertisol and Mollisol soil.

\begin{tabular}{|c|c|c|c|c|c|c|}
\hline Campaña/suelo & $07 / 08$ & $08 / 09$ & $09 / 10$ & $10 / 11$ & $11 / 12$ & $12 / 13$ \\
\hline Molisol & Trigo/soja & Girasol & Soja $1^{\mathrm{a}}$ & Soja $1^{a}$ & Trigo/soja & Girasol \\
\hline Vertisol & Maíz & Soja $1^{\mathrm{a}}$ & Trigo/soja & Maíz & Soja $1^{a}$ & Trigo/soja \\
\hline
\end{tabular}




\section{Variables estudiadas}

El análisis de la actividad respiratoria (AR) se realizó por la medición de las condiciones hipobáricas en la atmósfera de un recipiente cerrado debidas al consumo de $\mathrm{O}_{2}$ de los organismos del suelo. El $\mathrm{CO}_{2}$ producido se absorbe en un álcali para evitar la potencial interferencia de este gas en la medición de presión. Se empleó equipo Oxitop OC110 que brinda resultados de miligramos de $\mathrm{O}_{2}$ consumido. Por medio de cálculos se transforma esta medición a contenido de C$\mathrm{CO}_{2}$ (DIN 19737).

El carbono de la biomasa microbiana (CBM) se determinó por la técnica de fumigación-extracción (Vance et al. 1987; ISO 1997). La determinación incluye una fumigación de las muestras con cloroformo y una posterior extracción con sulfato de potasio. El C presente se evaluó mediante oxidación con dicromato de potasio y ácido sulfúrico. El cociente metabólico $\left(\mathrm{qCO}_{2}\right)$ es la cantidad de $\mathrm{CO}_{2}$ respirado por unidad de masa microbiana en un determinado tiempo. Se lo calculó como la relación entre la respiración y el CBM, y se expresó en $\mu \mathrm{g}$ $\mathrm{C}-\mathrm{CO}_{2} \times \mu \mathrm{g}$ de $\mathrm{CBM}^{-1} \times \mathrm{h}^{-1}$ (Anderson 2004). La capacidad de aporte de $\mathrm{N}$ del suelo (PMNIA) se realizó por determinación del $\mathrm{N}$ mineralizado en incubaciones anaeróbicas (Waring and Bremmer 1964), que consistió en una incubación en condiciones anaeróbicas durante siete días y posterior determinación del $\mathrm{N}$ mineralizado por destilación con óxido de $\mathrm{Mg}$. Las variables CBM y PMN-IA

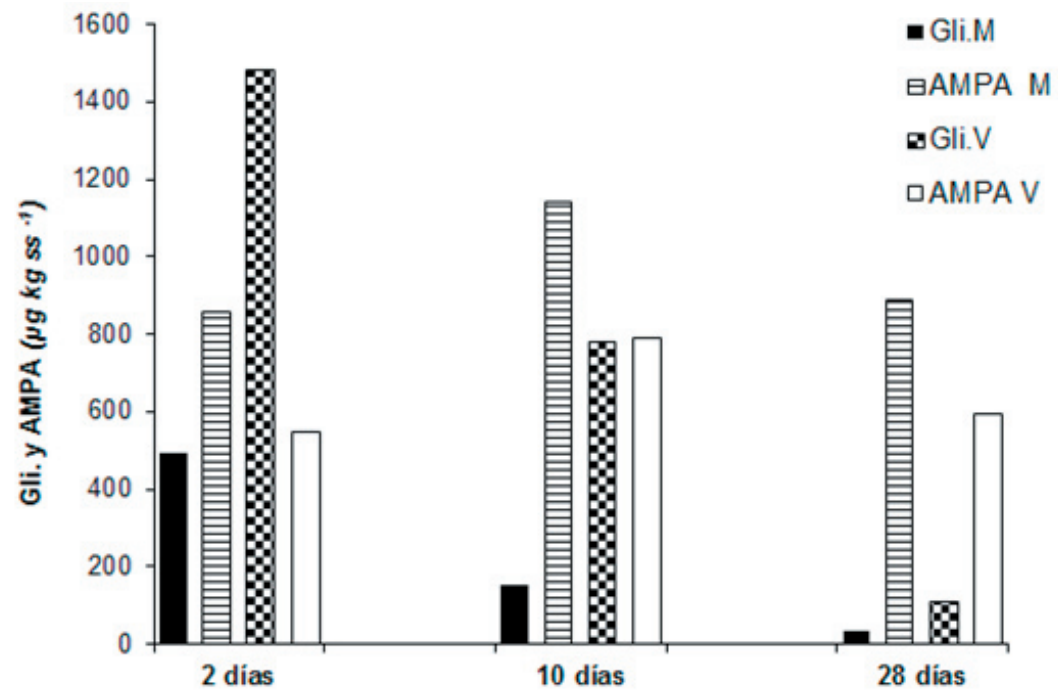

se determinaron antes de la aplicación del herbicida (inicio), a los 2 y 28 días posteriores a la aplicación. Las variables $\mathrm{AR}$ y $\mathrm{qCO}_{2}$ se midieron a los 2 y 28 días posteriores a la aplicación de glifosato.

La residualidad de glifosato y ácido aminometilfosfónico (AMPA) fue cuantificada por cromatografía líquida y espectrometría de masas. Estas determinaciones se realizaron sobre una sola muestra de cada tratamiento con aplicación de glifosato, cada una de ellas compuesta por porciones de suelo provenientes de las 3 repeticiones, por lo tanto no se contó con repeticiones para AMPA. Los análisis se realizaron en el laboratorio de análisis de pesticidas de la EEA Balcarce del INTA (SAGPyA). Los momentos de evaluación de residualidad de glifosato y AMPA fueron a los 2, 10 y 28 días posteriores a la aplicación del herbicida.

\section{Análisis estadístico}

Se llevó a cabo un análisis factorial (ANOVA) para evaluar la interacción entre el tratamiento aplicado y el suelo donde se realizó, para las variables CBM y PMN-IA, en los dos períodos de tiempo evaluados (2 y 28 días) (prueba de Duncan, $P \leq 0.05)$. Se aplicó el paquete estadístico infostat (Di Renzo et al. 2013).

\section{Resultados}

El glifosato mostró una residualidad baja en ambos suelos, disminuyendo su concentración 28 días después de su aplicación. Sin embargo, 

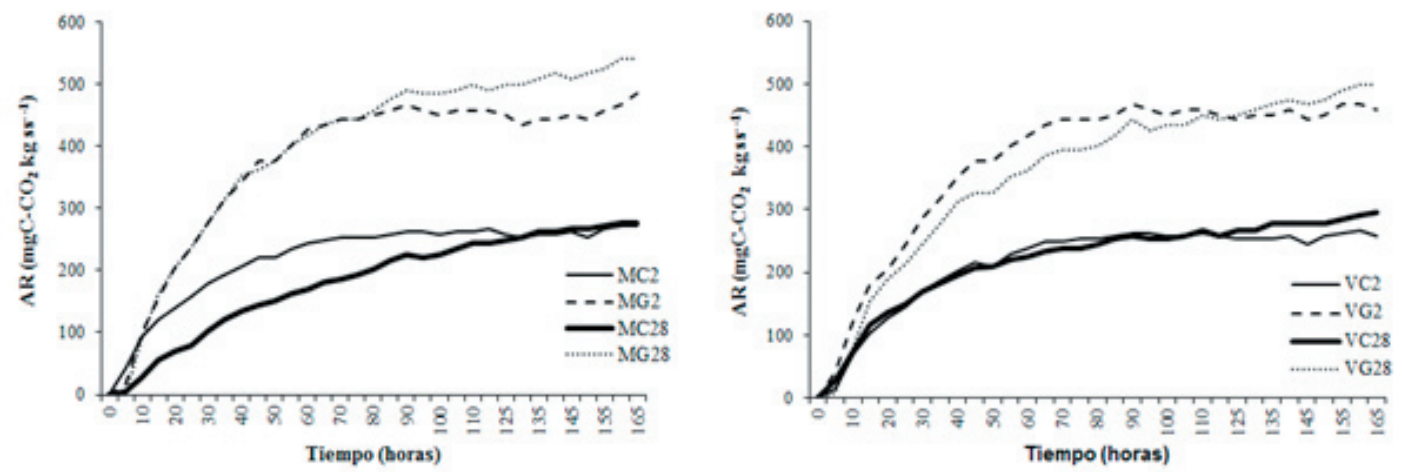

Figura 2.Actividad respiratoria acumulada (AR) en suelos Molisol y Vertisol sin aplicación de glifosato, medida a los 2 días (MC2 y VC2) y a los 28 días (MC28 y VC28) posteriores a la aplicación, y con aplicación de glifosato, medida a los 2 días (MG2 y VG2) y a los 28 días (MG28 y VG28) posteriores a la aplicación.

Figure 2. Respiratory activity (RA) in Mollisol and Vertisol soils, without application of glyphosate, measured after 2 days of application (MC2 and VC2) and after 28 days of application (MC28 and VC28), and soil with application of glyphosate, measured after 2 days (MG2 and VG2) and after 28 days of application (MG28 and VG28).
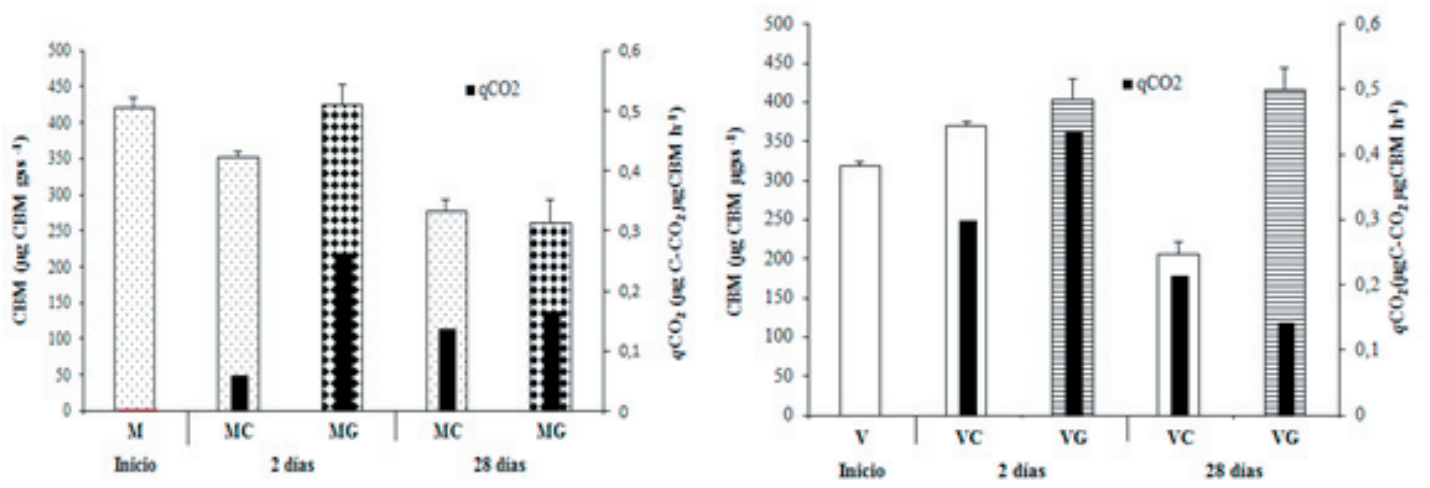

Figura 3. Carbono de la biomasa microbiana $(\mathrm{CBM})$ y cociente metabólico ( $q \mathrm{CO} 2)$ en suelos Molisol y Vertisol, al inicio (M y V), sin aplicación de glifosato (MC y VC) y con aplicación de glifosato (MG y VG).

Figure 3. Microbial biomass carbon (MBC) and metabolic quotient ( $q \mathrm{CO} 2)$ in Mollisol and Vertisol soils, at initial situation (M and V), without application of glyphosate (MC and VC ) and with glyphosate application (MG and VG).
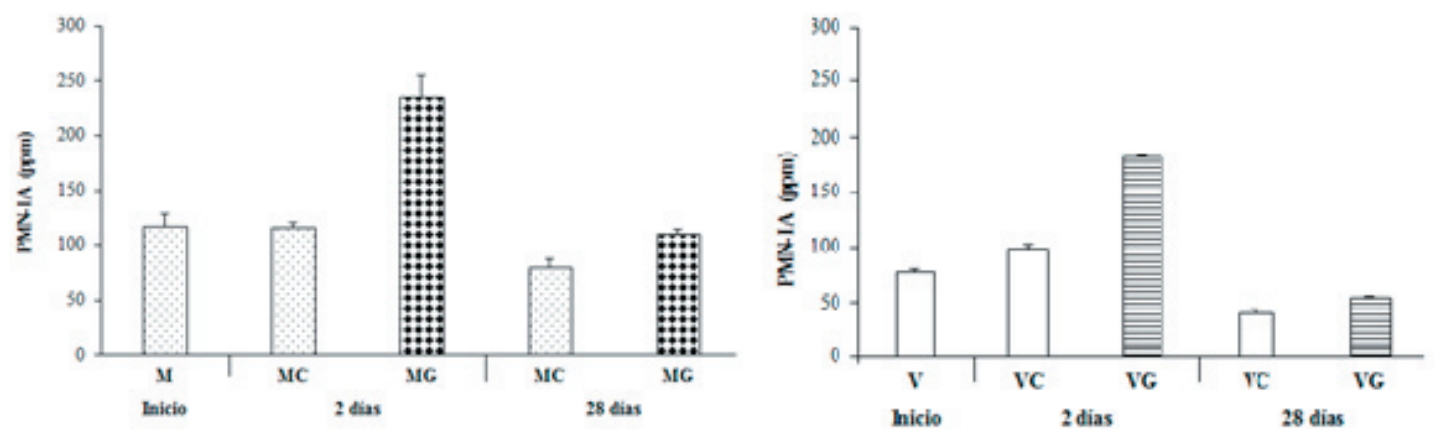

Figura 4. Potencial de mineralización de nitrógeno por incubaciones anaeróbicas (PMN-IA) en suelos Molisol y Vertisol, al inicio (M y V), sin aplicación de glifosato (MC y VC) y con aplicación de glifosato a los dos días (MG y VG).

Figure 4. Potential nitrogen mineralization for anaerobic incubations (PMN-IA) in Mollisol and Vertisol soils, at initial situation (M and V), without application of glyphosate (MC and VC) and with glyphosate application (MG and VG). 
Tabla 2. Cuadro de ANOVA para la variable C de la biomasa microbiana (CBM) a los 2 y 28 días posteriores a la aplicación del glifosato.

Table 2. ANOVA results for microbial biomass C (CBM) at 2 and 28 days after glyphosate application.

\begin{tabular}{lrrrrrrrrrr}
\hline \multicolumn{1}{c}{ F.V. } & \multicolumn{1}{c}{ SC } & g.l. & \multicolumn{1}{c}{ CM } & \multicolumn{1}{c}{ F } & \multicolumn{1}{c}{$P$} & \multicolumn{1}{c}{ SC } & g.l. & \multicolumn{1}{c}{ CM } & \multicolumn{1}{c}{ F } & \multicolumn{1}{c}{$P$} \\
\hline Modelo & 9630.57 & 3 & 3210.19 & 2.23 & 0.1616 & 71604.5 & 3 & 23868.17 & 10.85 & 0.0034 \\
Suelo & 7.52 & 1 & 7.52 & 0.01 & 0.9441 & 4977.61 & 1 & 4977.61 & 2.26 & 0.171 \\
Tratamiento & 8656.44 & 1 & 8656.44 & 6.02 & 0.0397 & 27821.07 & 1 & 27821.07 & 12.64 & 0.0074 \\
Suelo*Trat. & 966.61 & 1 & 966.61 & 0.67 & 0.4359 & 38805.81 & 1 & 38805.81 & 17.63 & 0.003 \\
$\quad$ Error & 11494.78 & 8 & 1436.85 & & & 17605.36 & 8 & 2200.67 & & \\
\multicolumn{1}{r}{ Total } & 21125.35 & 11 & & & & 89209.86 & 11 & & & \\
\hline
\end{tabular}

los contenidos de AMPA fueron altos durante todo el período estudiado en ambos suelos (Figura 1). Las concentraciones de glifosato fueron mayores en los suelos Vertisoles y las de AMPA mayores en los Molisoles.

La aplicación de glifosato aumentó la respiración (AR) en ambos suelos y tendió a aumentar el CBM y el PMN-IA, pero estas diferencias fueron en general no significativas, tanto a los dos como a los 28 días y en ambos suelos (Figuras 2, 3 y 4). Los contenidos medios de CBM fueron $415 \mu \mathrm{g} \mathrm{CBM} \mathrm{gss}{ }^{-1}$ para aquellos suelos con aplicación de glifosato y de $361 \mu \mathrm{g}$ $\mathrm{CBM} / \mathrm{g}$ de suelo seco para los tratamientos control (Figura 3). Solo a los 28 días y en el Vertisol, el valor registrado en el tratamiento con glifosato fue $416 \mu \mathrm{g} \mathrm{CBM} / \mathrm{g}$ de suelo seco y significativamente mayor (Tabla 2) que los demás tratamientos. A los 2 días, el $\mathrm{qCO}_{2}$ tomó valores de 0.263 para MG y 0.437 para VG, y de 0.06 en MC y 0.299 en VC. A los 28 días, los valores de $\mathrm{qCO}_{2}$ fueron 0.165 para MG y 0.143 para VG, y 0.136 para MC y 0.214 para VC (Figura 3). En este trabajo, el PMN-IA no mostró diferencias significativas entre los tratamientos (Tabla 3) para los dos momentos evaluados.

\section{Discusión}

En este trabajo se observó una mayor residualidad de glifosato en el tiempo en el suelo Vertisol, lo cual pudo haberse debido al mayor porcentaje de arcillas en comparación con el suelo Molisol (Figura 1). Estos resultados coinciden con el informe realizado por la Universidad Nacional del Litoral (2010) y Aparicio et al. (2013), quienes mencionan que uno de los factores que más afecta el tiempo de permanencia de las concentraciones de glifosato y AMPA en el suelo son las características físico químicas de los mismos y la adsorción sobre partículas minerales, más que los días desde la aplicación.
Los trabajos de Tejada (2009) y Kloosterman et al. (2010) también corroboran este aspecto relacionado a la adsorción del herbicida a las partículas minerales de arcilla del suelo. La degradación del glifosato depende de la comunidad microbiana presente en el suelo y posiblemente antes de la aplicación de glifosato la misma haya sido influenciada no sólo por diferencias en las propiedades fisco-químicas de los suelos (Lauber et al. 2008), sino también por las rotaciones de los diferentes cultivos antecesores en cada lote (ver secuencias de cultivos en la Tabla 1).

La AR fue mayor en los tratamientos con aplicación de glifosato (Figura 2a y 2b) posiblemente porque el glifosato puede ser usado por los microorganismos como una fuente de sustrato carbonado disponible con facilidad, lo cual estimularía el metabolismo respiratorio. Nuestros resultados concuerdan con los de Al Rajab et al. (2010), quien monitoreó la mineralización del glifosato y del AMPA en distintos tipos de suelos y encontró una inmediata y rápida tasa de degradación después de la aplicación y la ausencia de una fase de latencia, lo cual indica que la microflora del suelo tendría el sistema enzimático necesario para poder degradar estos compuestos. Bórtoli et al. (2012) y Araujo et al. (2003), cuando estudiaron las tasas de respiración en suelos con aplicación de glifosato, también encontraron que en aquellos tratados hubo un aumento de la AR hasta el día 30 de incubación, lo que pudo estar asociado a las cantidades de sustratos lábiles, y que los menores valores fueron observados a partir del día 55.

Los contenidos de CBM tendieron a ser mayores en los suelos con aplicación de glifosato en comparación con los controles, aunque los cambios dependieron del suelo donde se aplicó el herbicida (Figura 3). Esto muestra que para la dosis utilizada no hubo 
efecto germistático del glifosato sobre la masa de microorganismos y que el herbicida se pudo haber utilizado como fuente de energía y de carbono para el protoplasma microbiano. En suelos con labranzas conservacionistas, Panettieri et al. (2013) observaron que a los 18 días posteriores a la aplicación del glifosato se incrementaron los contenidos de CBM, con diferencias significativas entre los tratamientos con y sin aplicación de glifosato y sin diferencias entre tratamientos a partir del día 57.

El $\mathrm{qCO}_{2}$ nos permite mostrar mejor el destino de la energía con respecto a la masa de microorganismos. A los 2 días, los valores encontrados en los suelos con aplicación del herbicida presentaron cambios en la fisiología de los microorganismos, posiblemente debido a que las poblaciones microbianas fueron alteradas en su metabolismo (mayor AR) por la incorporación del agroquímico. Esto demuestra la rápida degradación asociada a una menor residualidad de glifosato a los 10 y 28 días (Figura 1). A su vez, esto puede indicar una menor eficiencia metabólica por parte de los microorganismos. Sterren et al. (2013) encontraron que a 5 días de aplicado el herbicida en un Vertisol de la provincia de Entre Ríos hubo un aumento de 13\% del CBM con respecto al suelo control y que, a diferencia de este ensayo, el $\mathrm{qCO}_{2}$ fue menor en el suelo con aplicación de glifosato, lo cual no fue lo esperable, y lo asociaron a la presencia de organismos menos eficientes en el C respirado por unidad de biomasa microbiana. Cuando trabajó en un Argiudol vértico, Gómez et al. (2009) también encontraron incrementos del $\mathrm{qCO}_{2}$ a los 4 días de aplicado el glifosato en dosis altas (3.84 L/ha de principio activo), con diferencias significativas con respecto al control, y sin diferencias entre ambos a los 45 días de incubación.

A los 28 días, los valores de $\mathrm{qCO}_{2}$ presuponen una estabilización de las tasas de respiración, explicable en relación al tiempo de incubación trascurrido desde la aplicación del herbicida.
En este período, los valores de $\mathrm{qCO}_{2}$ fueron similares en ambos suelos, pero en Vertisol estuvo asociado a mayores contenidos de CBM, lo que estaría relacionado con los distintos grupos de microorganismos del suelo. Bórtoli et al. (2006) encontraron aumentos significativos de la biomasa microbiana entre los días 15 y 30 de incubación, y también mencionaron que algunos grupos taxonómicos fueron estimulados en desmedro de otros. Esta característica presupone cambios en las comunidades microbianas, incluso que quizás los hongos (con mayor masa microbiana y menor AR) comenzaron a predominar en respuesta a cambios que afectan algunas de sus funciones.

El PMN-IA está asociado a los procesos metabólicos de los microorganismos en el suelo y, en especial, a la mineralización del $\mathrm{N}$ de compuestos orgánicos. En este trabajo se encontró que tendió a ser mayor en los suelos con aplicación de glifosato, aunque sin diferencias significativas entre los tratamientos (Tabla 3). Esto tal vez estuvo asociado a las mayores actividades degradativas (AR). Estos contenidos se podrían asociar a los contenidos de nitrógeno de la biomasa (NBM) para observar el destino del $\mathrm{N}$ en los pooles del suelo, aunque diversos autores han encontrado comportamientos distintos de la variable NBM en suelos con y sin aplicación de glifosato (Gómez et al. 2009; Panettieri et al. 2013; Wardle and Parkinson 1990).

El presente trabajo puso en evidencia que la aplicación del herbicida glifosato impactó de manera diferente en los Molisoles y en los Vertisoles. Mientras que en los Molisoles las poblaciones microbianas no fueron afectadas, en los Vertisoles las poblaciones, medidas por su contenido de $\mathrm{C}$, aumentaron acompañando la mayor residualidad del herbicida en estos suelos. Sin embargo, resultaría de interés investigar si los grupos microbianos cambian en relación a los períodos de tiempo postaplicación del herbicida.

\section{Referencias}

Al Rabaj, A., and M. Schiavon. 2005. The retention of gliphosate in differents three agricultural soils. 35th Congress of French Group of Pesticides. Comunicación oral.

Al Rajab, A. J., and M. Schiavon. 2010. Degradation of C-Glyphosate and aminomethylphosphonic acid (AMPA) in three agricultural soils. J Environ Sci 22(9):1374-1380.

Alexa, E., M. Bragea, R. Sumalan, A. Lazureanu, M. Negrea, and S. Iancu. 2009. Dynamic of glyphosate mineralization in different soil types. Romanian Agricultural Research 26:57-60.

Anderson, T. H. 2004. Microbial eco-physiological indicators to asses soil quality. Agric Ecosyst Environ 98:285-293. Anderson, T. H., and K. H. Domsch. 2010. Soil microbial biomass: The eco-physiological approach. Soil Biol Biochem 
42:2039-2043

Aparicio, V. C., E. De Gerónimo, D. Marino, J. Primost, P. Carriquiriborde, and J. Costa. 2013. Environmental fate of gliphosate and aminomethylphosphonic acid in surface waters and soil of agricultural basins. Chesmosphere 93: 1866-1873.

Araujo, A. S. F., R. T. R. Monteiro, and R. B. Abarkeli. 2003. Effect of glyphosate on the microbial activity of two Brazilian soils. Chemosphere 52:799-804.

Benintende, S., M. Benintende, and J. De Battista. 2005 Relaciones entre indicadores biológicos en rotaciones con arroz en suelos Vertisoles. CD ROM - V REBIOS. 6 al 8 de julio.

Biederbeck, V. O., R. P. Zentner, and C. A. Campbell. 2005. Soil microbial populations and activities as influenced by legume green fallow in a semiarid climate. Soil Biol Biochem 37(10):1775-1784.

Bittencuort Barreto, P., E. Forestieri, A. Gama-Rodríguez, N. De Barros, and S. Fonseca. 2008. Actividade microbiana, carbono e nitrógeno da biomassa microbiana emplantaçôes de eucalipto, emseqüência de idades. R Bras Ci Solo 32: 611-619.

Bollero, G. 2000. Soil Quality Assessment. XVII Congreso Argentino de la Ciencia del Suelo. Mar del Plata.

Bórtoli, P. V., R. A. Verdenelli, C. Conforto, S. Vargas Gil, and J. M. Meriles. 2012. Efectos del herbicida glifosato sobre la estructura y el funcionamiento de comunidades microbianas de dos suelos de plantaciones de olivo. Ecología Austral 22:33-42.

Calviño, P. A., and H. E. Echeverría. 2003. Incubación anaeróbica del suelo como diagnóstico de la respuesta a nitrógeno del maíz bajo siembra directa. Ciencia del suelo 21(1):24-29.

De Santana, H., L. R. M. Toni, L. O. de B. Benetoli. C. T. B. V. Zaia, M. Rosa Jr., and D. A. M. Zaia. 2006. Effect in glyphosate adsorption on clays and soils heated and characterization by FT-IR spectroscopy. Geoderma 136:738-750.

Di Rienzo, J. A., F. Casanoves, M. G. Balzarini, L. González, M. Tablada, and C. W. Robledo. 2013. InfoStat, versión 2013, Grupo InfoStat, FCA, Universidad Nacional de Córdoba, Argentina. URL: www.infostat.com.ar

DIN 19737: 2001-04. Soil quality - Laboratory methods for determination of microbial soil respiration. Kap. 4.1.2.1a, S. 9. Beuth Verlag, Berlin. 2001. Pp. 14.

Dion, H. M., Harsh, J. B., and H. H. Hill. 2001. Competitive sorption between glyphosate and inorganic phosphate on clay minerals and low organic matter soils. J Radioanal Nucl Chem 249:385-390.

Dos Santos Afonso, M. 2010. Comportamiento del glifosato en la interfaseagua:sólido. Una aproximación al estudio en suelos. En: Aspectos ambientales del uso de glifosato. INTA Balcarce. Pp. 114.

Echeverría, H. E., N. F. San Martín, and R. Bergonzi. 2000. Métodos rápidos de estimación de nitrógeno potencialmente mineralizable en suelos. Ciencia del Suelo 18(1):9-16.

Feng, J. C., and D. G. Thompson. 1990. Fate of glyphosate in a Canadian forestwatershed. 2. Persistence in foliage and soils. Journal of Agricultural and Food Chemistry 38(4):1118-1125.

Ferreira, E. A., J. B. Santos, A. A. Silva, L. Vargas, and M. R. Reis. 2006. Glyphoste application for Italian ryegrass biotype control and impactoon soil microbiota. Planta Daninhia 24:573-578.

Gerritse, R. G., Beltrán, J., and F. Hernández.1996.Adsorption of atrazine, simazine, and glyphosate in soils of the Gnangara Mound, Western Australia. Aust J Soil Res 34:599-607.

Gimsing, A. L., O. K. Borggaard, and M. Bang. 2004. Influence of soil composition on adsorption of glyphosate and phosphate by contrasting Danish surface soils. Eur J Soil Sci 55:183-191.

Glass, R. L. 1987. Adsorption of glyphosate by soils and clay minerals. J Agric Food Chem 35:497-500.

Gómez, E., L. Ferreras, L. Lovotti, and E. Fernández. 2009. Impact of glyphosate application on microbial biomass and metabolic activity in a Vertic Argiudoll from Argentina. European Journal of Soil Biology 45:163-167.

Informe acerca del grado de toxicidad del glifosato. 2010. Universidad Nacional del Litoral. ISBN: 987-657-506-6. Pp. 240.

ISO 10381-6. 1993. Soil quality. Sampling. Part 6: Guidance on the collection, handling and storage of soil for the assessment of aerobic microbial processes in the laboratory.

ISO 14240-2. 1997. Soil quality: determination of soil microbial biomass - part 2: fumigation-extraction method. Geneva: International Organization for Standardization.

Kloosterman, M. E., J. L. Costa, M. Zelaya, and F. Bedmar. 2010. Estudio de la adsorción del glifosato en un Argiudol típico del Sudeste Bonaerense. XXII Congreso Argentino de la Ciencia del Suelo. Rosario, Argentina. Actas en CD ROM. ISBN 978-987-24771-3-4.

Lauber, C. L., M. S. Strickland, M. A. Bradford, and N. Fierer. 2008. The influence of soil properties on the structure of bacterial and fungal communities across land-use types. Soil Biol Biochem 40:2407-2415

Lerch, T. Z., M. F. Dignac, N. Nunan, G. Bardoux, E. Barriuso, and A. Mariotti. 2009. Dynamics of soil microbial populations involved in 2,4-D biodegradation revealed by FAME-based table Isotope Probing. Soil Biol Biochem 41(1):77-85.

Morillo, E., T. Undabeytia, C. Maqueda, and A. Ramos. 2002. The effect of dissolved glyphosate upon the sorption of copper by three selected soils. Chemosphere 47:747-752.

Nakatani, A. S., M. Ferreira, S. Fernandes, R. Aparecida de Souza, A. Pereira da Silva, F. Bueno dos Reis-Junior, I. Carvalho Mendes, and M. Hungría. 2014. Effects of the glyphosate-resistance gene and of herbicides applied to the soybean crop on soil microbial biomass and enzimes. Field Crops Res 162:20-29.

Panettieri, M., L. Lázaro, R. López Garrido, J. M. Murillo, and E. Madejón. 2013. Glyphosate effect on soil biochemical 
properties under conservation tillage. Soil \& Tillage Res 133:16-24.

Pessagno, R., R. M. Torres Sánchez, and M. Afonso. 2008. Glyphosate behavior at soil and mineral - water interfaces. Environ Pollut 153:53-59.

Piccolo, A., G. Celano, M. Arienzo, and A. Mirabella. 1994. Adsorption and desorption of glyphosate in some European soils. J Environ Sci. Health, Part B, v.6. Pp. 1105-1115.

Plan Mapa de Suelo. 1998. Cartas de Suelo República Argentina. Provincia de Entre Ríos. Departamento Paraná. Acuerdo complementario Convenio INTA- Gob. Entre Ríos. Serie de Relevamiento de Recursos Naturales № 17. ISSN-0325-9099. Pp. 114.

Plan Mapa De Suelo. 2001. Cartas de Suelo República Argentina. Provincia de Entre Ríos. Departamento Nogoyá. Acuerdo complementario Convenio INTA- Gob. Entre Ríos. Serie de Relevamiento de Recursos Naturales № 20. ISSN-0325-9099. Pp. 292.

Primost, J., D. Marino, V. Aparicio, J. Costa, A. Ronco, and P. Carriquiriborde. 2012. Comportamiento ambiental del glifosato en una microcuenca de Entre Ríos. Universidad Nacional de La Plata. Resultados no publicados.

Soil Survey Staff. 2010. United States Department of Agriculture. Keys to Soil Taxonomy. Eleventh Edition. Pp. 337.

Sparks, D. L. 2003. Environmental soil chemistry. Academic press. Inc. Londres. UK.

Sterren, M., W. Uhrich, V. Ormaechea, and S. Benintende. 2013. Indicadores ecofisiológicos en suelos molisoles y vertisoles con aplicación de glifosato. ACTAS CD ROM. IX Reunión Nacional Científico-Técnica de Biología de Suelos. I Congreso Nacional de Biología Molecular de Suelos. Santiago del Estero.

Tejada, M. 2009. Evolution of soil properties after addition of glyfosate, diflufenican and glyphosate+diflufenicanher bicides. Chemosphere 76:365-373.

Turco, R. F., A. C. Kennedy, and M. D. Jawson. Microbial indicators of soil quality. In: Doran JW, Coleman D, Bezdicek D, Stewart B, editors. 1994. Defining soil quality for a sustainable environment. SSSA Special Publication 35. Madison, Wisconsin, USA. Pp. 73-90.

University Of Hertfordshire. 2010. Pesticide Database. URL: https:/sitem.herts.ac.uk/aeru/footprint/es/Reports/ 373.htm

Vance, E., P. C. Brookes, and D. S. Jenkinson. 1987. An extraction method for measuring soil microbial biomass C. Soil Biol Biochem 19:703-707.

Wardle, D. A., and D. Parkinson. 1990. Effects of three herbicides on soil microbial activity and biomass. Plant Soil 122:21-28.

Vereecken, H. 2005. Mobility and leaching of glyphosate: a review. Pest Management Science 61(12):1139-1151.

Waring, S. A., and J. M. Bremner. 1964. Ammonium production in soil under waterlogged conditions as an index of nitrogen availability. Nature 201:951-952.

Wiren-Lehr, S., D. Komoba, and W. E. Glabgen.1997.Mineralization of [14C] glyphosate and its plant-associated residues in arable soils originating from different farming systems. Pesticide Sci 51(4):436-442. 